Necati Iyikan

\title{
Die Ereignisse vom 11. September 2001 in den USA und deren politische Bedeutung für die Türkei
}

Die Ereignisse vom 11. September 2001 haben die Prioritäten der Weltpolitik dramatisch verändert: Der Islam und insbesondere der islamistisch-fundamentalistisch motivierte Terrorismus stehen seither im Fokus des Interesses. Die Ursachen für den Zulauf, den der Terrorismus im Islam seit Jahren verzeichnet, sind sehr komplex und ihre historische Einordnung eine andauernde Herausforderung. Der vorliegende Aufsatz will dazu einen Beitrag leisten und dabei insbesondere zeigen, welche Rolle die islamisch geprägte, aber laizistische Türkei als Mittler zwischen der christlich geprägten westlichen Welt und der islamischen Kultur spielen könnte.

Ein wesentliches mit den Anschlägen vom 11. September verfolgtes Ziel war es, den Einfluss der USA in der islamischen Welt zurückzudrängen. Dieses Ziel wurde nicht nur nicht erreicht: Spätestens mit dem Angriff auf den Irak im Jahre 2003 haben die USA im Gegenteil unmissverständlich deutlich gemacht, dass sie ihren Einfluss in der Region militärisch zu verteidigen fest entschlossen sind. Der Terrorgefahr wollen sie demnach vor allem dadurch begegnen, dass sie in der islamischen Welt mit militärischer Gewalt Demokratien nach westlichem Vorbild installieren. Mittlerweile sollte allerdings deutlich geworden sein, dass es eine Lösung des Terrorproblems mit militärischen Mitteln allein nicht geben kann. Im Gegenteil erleben islamistische Terrororganisationen dank der militärischen Interventionen des Westens Zulauf auch aus zuvor eher gemäßigten Kreisen. Statt auf die militärische Karte zu setzen wäre es deshalb nötig, neue Strategien der Verständigung zu entwickeln. Die Türkei könnte dazu einen wichtigen Beitrag leisten. Mit ihrer historischen Erfahrung wäre sie ein prädestinierter Mittler zwischen der islamischen Welt und dem Westen.

\section{Der 11. September}

Die Gefahr des islamischen Fundamentalismus, für den der Islam aber selbst nicht einstehen kann, bewegt sich innerhalb einer vom Westen, aber auch von islamischen Ländern nicht erkennbaren Grenze. Nach dem 11. September drängten sich zuvor sträflich vernachlässigte Fragen in den Vordergrund: Welche Ursachen hat der zunehmende islamische Fundamentalismus und wie kann man ihnen begegnen? Warum gehen (vor allem junge Leute) als Selbstmordattentäter in den Tod? Nicht nur im Westen hatte man diese Fragen bis dahin sträflich vernachlässigt: Auch in den islamischen Ländern war man ihnen auf politischer Ebene weigehend mit Gleichgültigkeit begegnet. Im Westen galt das Motto: Solange die wirtschaftlichen 
und politischen Interessen nicht in Gefahr sind, bedarf der islamische Fundamentalismus keiner größeren Aufmerksamkeit. Das Motto in den islamischen Ländern hieß: Solange fundamentalistische Bewegungen die bestehende Herrschaftsordnung nicht gefährden, besteht kein Handlungsbedarf. Hier wie dort stand die vom islamischen Fundamentalismus ausgehende Gefahr also nicht sonderlich weit oben auf der politischen Tagesordnung.

\subsection{Die Lehre aus dem 11. September}

Die Taliban in Afghanistan und deren Führer Osama Bin Laden haben die Anschläge in den USA 2001 zu verantworten. Die Antwort auf die Frage: »Was kann man aus dem 11. September ableiten?«, wird die künftige Sicherheit in der Welt bestimmen. Die Vorschläge etwa eines Michael O'Hanlon, einer der wichtigsten Stimmen in der amerikanischen Militärpublizistik, der daraus lediglich die Forderung ableitete, die militärische Schlagkraft der USA zu stärken, erwecken den Eindruck, dass man den tatsächlichen Ernst der Lage noch immer nicht wahrnimmt und aus dem 11. September nicht viel gelernt hat. Aus seinem Werk »Neue Herausforderungen für die Verteidigungspolitik der USA « erfährt man, dass das Verteidigungsbudget der USA ein Drittel der weltweiten Rüstungsausgaben ausmacht. Die Erklärung, warum die USA eine solche Schlagkraft brauchen, hält der Verfasser des vorliegenden Aufsatzes für wenig überzeugend: »[...] Wie sehr die USA und der Westen diese Schlagkraft benötigen, haben die Ereignisse seit dem 11. September 2001 gezeigt. «1 Die beste Technologie, die modernsten Kommunikationsmöglichkeiten und der mächtigste Militärapparat sind nicht in der Lage gewesen, einen Terrorakt wie den vom 11. September $2001 \mathrm{zu}$ verhindern. Die USA hatten kaum Schwierigkeiten, die militärische Infrastruktur der Taliban in Schutt und Asche zu legen. Die amerikanischen und britischen Luftangriffe gegen die Taliban in Afghanistan nach dem 11. September 2001 erbrachten für die Weltöffentlichkeit kein fassbares Ergebnis. Die Bodentruppen, deren Ziel es war, den Terrorchef Osama Bin Laden zu fassen, hatten dabei ebenso wenig Erfolg. Man muss demnach den islamischen Terror auf einem anderen Weg bekämpfen. Einer der Lösungsvorschläge ist die Verbesserung der Ausbildung der Bevölkerung in den islamischen Ländern. Sicherlich würde sich eine solche durchaus positiv auf die Lebensqualität der Menschen auswirken. Aber wäre diese Verbesserung eine Maßnahme gegen den Fundamentalismus? In Afghanistan beispielsweise können rund 80 Prozent der Menschen weder lesen noch schreiben. ${ }^{2}$ Angenommen, das Problem des Analphabetismus in

1 Michael O'Hanlon, »Defense Policy Choices for the Bush Administration 2001-05, Washington D.C.: The Brookings Institution 2001, (Stephen Bierling übersetzte den Titel >Neue Herausforderungen für die Verteidigungspolitik der USA $)$ « in: Andreas Ufen, Postislamismus. Eine Auseinandersetzung mit der Zukunft der muslimischen Welt, in: Internationale Politik, Europa Archiv, Berlin Nr. 3 (2002), S. 57, 60, 61.

2 Axel Kuhlmann / Martin Agüera, "Die Hydra >Terrorismus< und ihre Auswirkungen auf die globale Sicherheitspolitik « in: Politische Studien, Zweimonatszeitschrift für Politik und Zeitgeschehen, München Nr. 381 (2002), S. 52. 
Afghanistan wäre gelöst, so bliebe es immer noch fraglich, ob die Menschen deshalb in der Lage wären einzuschätzen, ob eine islamische Fatwa mit der islamischen Lehre übereinstimmt oder nicht. Bin Laden verkündete im Februar 1998:

»[...]Alle Muslime haben die Pflicht, wo, wann und wie auch immer Amerikaner und ihre Alliierten zu töten. ${ }^{3}$ Dies entspricht wohl kaum dem wahren Islam. Gibt es einen Mechanismus, ein System oder ein religiöses Kontrollorgan, das die verkündeten Fatwas daraufhin überprüft, ob sie der Lehre des Islam auch tatsächlich entsprechen? Sicherlich, so etwas gibt es in den verschiedenen islamischen Ländern, allerdings werden diese »Kontrollorgane« von den jeweiligen Regierungen kontrolliert. Die Bevölkerungen ihrerseits haben nur Zugriff auf spärliche und einseitige Informationsquellen. Diese verkörpern dann die Propaganda oder die zum politischen Zweck manipulierte islamische Auffassung. In diesem Fall wäre also nichts gewonnen, wenn Muslime lesen und schreiben könnten.

Eine andere Maßnahme gegen den Fundamentalismus könnte die Verbesserung der wirtschaftlichen Lage in einigen islamischen Ländern sein. Allerdings kann diese kaum losgelöst von der gesamtwirtschaftlichen Lage in der Welt gesehen werden, was bedeutet, dass Entwicklungshilfe der westlichen Industriestaaten gefragter wäre denn je.

Dass es tatsächlich einen Zusammenhang zwischen Armut und Terror gibt, ist unbestreitbar. Doch wird religiöser Fanatismus im islamischen Raum bei weitem nicht nur durch Armut ausgelöst. Deutlich sichtbar wird dies an den handelnden Personen vom 11. September: Die Attentäter entstammten keinen armen Elternhäusern, waren gebildet, hatten Arbeit, Familien und Freundinnen. ${ }^{4}$ Sie entsprachen also nicht dem Bild, dass man sich von einem typischen Selbstmordattentäter macht, wie man ihn aus dem Krisenherd zwischen Israel und Palästina seit langem kennt. Das grundlegende Problem ist das politische System, in dem raffinierte Propagandisten junge Muslime zum Selbstmordanschlag bewegen können. Dieses System ermöglicht auch den Herrschern des betroffenen Landes jede denkbare Art des Missbrauchs von Religion. Die Aussage Atatürks: »Der Politiker, der zur Regierung die Religion braucht, ist ein Schwachkopf « ${ }^{5}$, kann man vor diesem Hintergrund vielleicht dahingehend ergänzen, dass ein solcher Politiker ein entschiedener Gegner der Religion sein muss, weil er sie zum politischen und wirtschaftlichen Zweck instrumentalisiert. Aus dieser Perspektive könnte man Atatürk wegen der Einführung des Laizismus in der Türkei nicht nur als türkischen, sondern auch als islamischer Revolutionär bezeichnen.

3 Kuhlmann / Agüera, Die Hydra >Terrorismus und ihre Auswirkungen auf die globale Sicherheitspolitik, aaO. (FN 2), S. 48.

4 Ahmed Rashid, Taliban. Afghanistans Gotteskrieger und der Dschibad. Aus dem Englischen von Harald Riemann, München 2001, S. 23.

5 Brigitte Moser / Michael W. Weithmann, Die Türkei. Nation zwischen Europa und dem Naben Osten, Regensburg 2002, S. 112. 


\subsubsection{Dialog als mögliches Mittel gegen Terror}

Wie aber könnte ein dauerhaft fruchtbarer Dialog zwischen dem Westen und der islamischen Welt initiiert werden? Ein wichtiger Aspekt wäre wohl die Befreiung des Islam von jedwedem politischen Einfluss. Dieses Ziel kann nur durch die kompromisslose Trennung von Religion und Politik erreicht werden, also in einem laizistischen Staat. Der Islam hat in einem laizistischen System auf die Gesetzgebung keinen Einfluss. Durch die Gründung der modernen türkischen Republik unter der Führung von Mustafa Kemal Atatürk wurde der Grundstein dafür 1923 gelegt. Wir müssen hier nicht auf die intensiven Diskussionen eingehen, inwieweit der Laizismus mit dem Islam überhaupt vereinbar ist, inwieweit die islamische Lehre mit dem westlichen Demokratieverständnis auf Dauer koexistieren kann und auch inwieweit die Säkularisierung bzw. der Laizismus in den anderen islamischen Ländern eingeführt werden kann. In dem hier verhandelten Zusammenhang genügt es, die Bedeutung der Türkei mit ihrem laizistischen System nach dem 11. September 2001 für den Dialog zwischen der christlichen und islamischen Welt darzustellen.

Die Türkei wird meist als geographische Brücke zwischen Europa und dem Nahen Osten definiert. Als einziges Land mit überwiegend islamischer Bevölkerung ist sie NATO-Mitglied und spielt eine unverzichtbare Rolle innerhalb des nordatlantischen Bündnisses in einer unruhigen Konfliktregion. Dieses laizistische System in der islamischen Welt wurde jedoch im Westen kaum zur Kenntnis genommen oder gar gewürdigt. Ein Beispiel dafür ist die Äußerung des deutschen CDU Europa-Politikers Peter Hintze zum geplanten Beitritt der Türkei zur EU. Nach ihm sei die EU vom christlichen Menschenbild geprägt, das die Einhaltung der Menschenrechte, die Gleichheit von Männern und Frauen und die soziale Marktwirtschaft garantiere. »Kein islamischer Staat unterstützt unsere Weltordnung. «6 Vorab ist festzuhalten, dass die Türkei kein islamischer, sondern ein laizistischer Staat ist. Diese Definition im Westen ist in der Gegenwart keine Seltenheit. Die Menschenrechtssituation in der Türkei mag zwar noch unbefriedigend sein, aber es sind im Zuge der angestrebten Reformen für den EU-Beitritt Fortschritte zu verzeichnen. Hinsichtlich der Gleichheit von Männern und Frauen sei aber folgender Hinweis angebracht: Die türkischen Frauen haben sehr früh eine rechtliche Gleichstellung erlangt, zunächst 1930 auf lokaler, dann in den Jahren 1931 und 1934 auf nationaler Ebene. Tatsächlich war die parlamentarische Repräsentation der türkischen Frauen in den Jahren 1935 bis 1946 mit am stärksten in der Welt. ${ }^{7}$ Zudem gibt es in der Türkei im Vergleich zu westlichen Ländern einen sehr hohen Anteil von Frauen in sozial hoch angesehenen akademischen Berufen und dabei auch gerade in den Berufen, die in den westlichen Ländern vorrangig den Männern vorbehalten sind. ${ }^{8}$ Diese Tatsache ist nicht nur als Folge der Umsetzung des Laizismus und der Demokratie in

6 Allgäner Zeitung, 20.12.2002.

7 Pembenaz Yorgun, Türkei. Staat und Gesellschaft, mit Beiträgen von Ron Ayres / Selahattin Doğan /Aydın Ender / Mehmet Salah / Turgut Taylan, Frankfurt 1987, S. 158.

8 Ursula Boos-Nünning / Petra Kappert / Faruk TMen, Türkei Sozialkunde (Wirtschaft, Beruf, Religion, Familie, Erziehung), für Türkeistudien, Hessen 1990, S. 142. 
der Türkei zu bewerten, sondern hat ihre Wurzel in der türkischen Geschichte, auf deren Hintergrund einzugehen nicht das Thema des Aufsatzes ist. Allerdings bedeutet dies nicht, dass diese durchaus positive Entwicklung fortgesetzt wurde. Ein Beispiel ist die dramatisch gesunkene parlamentarische Repräsentation der türkischen Frauen. Diese Zahl beträgt nur noch 24 von insgesamt 550 Abgeordneten in der Legislaturperiode im Jahr 20049', wobei die Tendenz im Westen eine umgekehrte Richtung nahm. Wichtig ist jedoch, dass die notwendige juristische Grundlage in der Verfassung gegeben ist und sie von der Mehrheit der Bevölkerung unterstützt wird, was in der Türkei der Fall ist.

\subsection{2 »Kampf der Kulturen«}

Hinsichtlich des weltweit diskutierten Schlagworts vom Kampf der Kulturen erfährt man bei Samuel P. Huntington, die Welt bestehe aus fünf bzw. sieben Kulturbereichen: dem sinischen, japanischen, hinduistischen, islamischen und westlichen Kulturkreis, gegebenenfalls zu ergänzen durch den lateinamerikanischen und den afrikanischen Kulturkreis. ${ }^{10}$ Wir wollen hier nicht der Frage nachgehen, ob Huntingtons These wirklich ernst zu nehmen oder nur als rhetorischer Versuch zu bezeichnen ist. Wesentlich sind die Beziehungen zwischen der christlichen und islamischen Welt, die sich nach dem 11 . September dramatisch verschlechtert haben. Nach dem amerikanischen und britischen Angriff auf den Irak im März 2003 droht die Lage zwischen den zwei Kulturen zu eskalieren, zumal die islamischen Bevölkerungen, insbesondere in einigen arabischen Ländern, diesen Krieg nicht als Krieg gegen das Regime Saddam Husseins auffassen, sondern als einzig gegen den Islam gerichtete Aggression. Saddam Hussein, der als aufgeklärter Herrscher angetreten war, entdeckte plötzlich, auch durch den ersten Golfkrieg 1991, der die Chancen für einen dauerhaften Frieden in Nahost deutlich verschlechtert hatte ${ }^{11}$, den Islam als wirksame Waffe und forderte die Muslime in aller Welt auf, sich dem heiligen Krieg am Golf gegen die »Ungläubigen « und »Schamlosen « anzuschließen ${ }^{12}$, womit er in die Fußstapfen seines Todfeindes Khomeini getreten war. ${ }^{13}$

Der Verfasser sieht in der derzeitigen politischen Konstellation eine potentielle Ausgangsbasis für die weltweite Eskalation gewalttätiger Auseinandersetzungen.

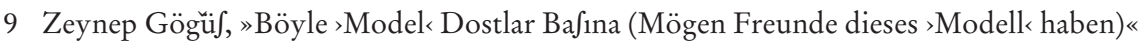
in: Hürriyet, 17.04.2004, (Zeynep Gögü ist türkische Journalistin ).

10 Dieter Senghaas, »Die fixe Idee vom Kampf der Kulturen« in: Blätter für deutsche und internationale Politik, Bonn 2 (1997), S. 215.

11 Sven Rohde, »Zwischen den Fronten. Ägypten, Marokko und Jordanien « in: Peter Scholl-Latour, Weltkrise Arabien. Allah, Blut und Öl-Hintergründe eines Konflikts, Gütersloh 1992, S. 138.

12 Ulrich Kienzle, »Ungezügelte Tyrannen. Syrien und Irak « in: Peter Scholl-Latour, Weltkrise Arabien. Allah, Blut und Öl-Hintergründe eines Konflikts, Gütersloh 1992, S. 94.

13 Peter Scholl-Latour, Weltkrise Arabien. Allah, Blut und Öl-Hintergründe eines Konflikts, Gütersloh 1992, S. 6. 
Verhindert werden könnte eine solche Eskalation durch einen effektiven Dialog zwischen der islamischen und christlichen Welt. Die laizistische, demokratische Türkei wäre als Mittler zwischen den Kulturen am ehesten in der Lage, einen solchen Dialog in Gang zu setzen und zu moderieren.

Samuel P. Huntington hält die Türkei für ein zerrissenes Land. Ihm zufolge muss ein solches Land drei Voraussetzungen erfüllen, um seine Identität erfolgreich neu definieren zu können: Die politische und wirtschaftliche Elite des Landes muss, erstens, diesen Schritt grundsätzlich unterstützen und aufrichtig begrüßen, zweitens muss die Öffentlichkeit bereit sein, diese Neudefinition ihrer Identität zumindest stillschweigend hinzunehmen, und drittens müssen die dominierenden Eliten der Wirtszivilisation, in den meisten Fällen also des Westens, bereit sein, den Konvertiten zu akzeptieren.

Die Türkei erfüllte Huntington zufolge viele Jahre lang zwei dieser drei Minimalanforderungen, die ein zerrissenes Land erfüllen muss, das seine Identität verändern will. Die Eliten der Türkei unterstützten ganz überwiegend diesen Schritt und die Öffentlichkeit fügte sich stillschweigend. Aber die Eliten der westlichen rezipierenden Zivilisation waren unempfänglich. ${ }^{14}$ Huntington verliert kaum ein Wort über den Laizismus in der Türkei und dessen möglicher Bedeutung für einen Dialog der Kulturen. Inwieweit man von dieser Türkei als »Chance« gegen den religiösen Fundamentalismus profitieren könnte, kann er demzufolge auch nicht diskutieren. Er meint zwar, die Türkei habe den Wunsch, den Iran und Saudi-Arabien an der Expansion ihres Einflusses und der Förderung des islamischen Fundamentalismus im Kaukasus und in Zentralasien zu hindern. Die Türken verstanden dagegen das von ihnen angebotene »türkische Modell« oder »die Idee der Türkei« - nämlich einen säkularen, demokratischen Staat mit einer Marktwirtschaft - als Alternative. ${ }^{15}$ Nach Huntington bezeichneten türkische Führer ihr Land gerne als Brücke zwischen den Kulturen. Der inzwischen verstorbene Staatspräsident Özal erklärte noch als Regierungschef, die Türkei beanspruche nunmehr die Brückenfunktion zwischen Europa und dem arabischen Orient. ${ }^{16}$ Die ehemalige Ministerpräsidentin Tansu Çiller äußerte 1993, die Türkei sei sowohl eine westliche Demokratie als auch ein Teil des Nahen Ostens und überbrücke somit physisch und philosophisch zwei Zivilisationen. Huntington zufolge ist eine Brücke jedoch ein künstliches Gebilde, das zwei Orte miteinander verbindet, selbst aber zu keinem von beiden gehört. Wenn die Führer der Türkei ihr Land eine Brücke nennen, bestätigen sie mit diesem Euphemismus, dass die Türkei ein zerrissenes Land ist. ${ }^{17}$

14 Samuel P. Huntington, Kampf der Kulturen. Die Neugestaltung der Weltpolitik im 21. Jabrbundert, München/Wien 1997, S. 218, 235.

15 Huntington, Kampf der Kulturen. Die Neugestaltung der Weltpolitik im 21. Jahrhundert, aaO. (FN 14), S. 231.

16 Peter Scholl-Latour, Das Schwert des Islam. Revolution im Namen Allabs, München 1990, S. 76.

17 Huntington, Kampf der Kulturen. Die Neugestaltung der Weltpolitik im 21. Jahrhundert, aaO. (FN 14), S. 235 f. 
Bei Huntington erfährt man über die islamische Kultur so gut wie nichts. Dies ist umso merkwürdiger, als er nicht etwa den Islamismus oder den islamischen Fundamentalismus zum Problem erklärt, sondern den Islam selbst, weil dieser, verglichen mit allen übrigen Kulturen, eine ganz andere Kultur darstellt. Er betont, dass sich muslimische Gesellschaften und Staaten an den kulturellen Bruchlinien der Welt als besonders gewalttätig erweisen: »Muslimische Kriegslust und Gewaltbereitschaft sind Ende des 20. Jahrhunderts eine Tatsache, die weder Muslime noch Nicht-Muslime leugnen können.« Huntingtons Darlegung unterstellt dem Islam implizit einen per se gewalttätigen Charakter. ${ }^{18}$ Demnach wären natürlich weder die Bereitschaft noch die Möglichkeit zum Dialog zwischen den zwei Kulturen gegeben. Huntington übersieht dabei die Tatsache, wie Dieter Senghaas treffend festgestellt hat, dass kulturelle und in aller Regel religiöse Faktoren selten am Ausgangspunkt einer Konflikteskalation durchschlagendes Gewicht besitzen. Vielmehr machen Verteilungskonflikte den Kern des Konfliktgeschehens aus. ${ }^{19}$ Auch wenn die Auseinandersetzungen an Kultur- und Konfessionsgrenzen ausbrechen, tragen sie primär politischen Charakter. ${ }^{20}$

$\mathrm{Ob}$ die Weltethos-Debatte, die auf die Begegnung der Kulturen und insbesondere auf den Dialog zwischen den maßgebenden Weltreligionen ausgerichtet is $\mathrm{t}^{21}$, dies auch wirklich tut, ist mehr als fraglich: »Kein Weltfrieden ohne Religionsfrieden, kein Religionsfrieden ohne Dialog zwischen den Religionen «22 klingt zwar gut, ein möglicher Religionsfrieden kann aber aus zwei Gründen zu keinem Weltfrieden führen:

Erstens: Die großen Religionen wie Christentum und Islam können in ihrem eigenen Glaubensraum keinen Frieden ermöglichen. Dies zeigen alleine schon die Meinungsunterschiede zwischen den drei Kirchen (katholische, evangelische und orthodoxe $)^{23}$ und die voneinander abweichenden islamischen Auffassungen in den islamischen Ländern sowie der acht Jahre (1980 - 1988) dauernde Krieg zwischen Iran und Irak.

18 Senghaas, Die fixe Idee vom Kampf der Kulturen, aaO. (FN 10), S. 216.

19 Senghaas, Die fixe Idee vom Kampf der Kulturen, aaO. (FN 10), S. 218, 220.

20 Uwe Halbach, "Der Kaukasus als Konfliktregion « in: Zeitschrift für internationale Fragen, Hamburg 4 (1997), S. 361.

21 Dieter Senghaas, Wohin driftet die Welt? Über die Zukunft friedlicher Koexistenz, Frankfurt am Main 1994, S. 115.

22 Hans Küng / Josef van Ess, Christentum und Weltreligionen. Islam, Gütersloh 1990, S. 194.

23 Ohne auf die Geschichte einzugehen, sei hier ein einfaches Beispiel aus dem Alltag genannt: Es handelt sich um Verhütungsmittel. Der Papst wendet sich gegen den Einsatz von Verhütungsmitteln. Die evangelische Kirche ist dagegen großzügiger. Es gibt aber auch Meinungsunterschiede in der katholischen Kirche zwischen Papst und unteren kirchlichen Hierarchien. Siehe dazu Kirchenpolitik, Bevölkerungspolitik, http:/ www.pro-liberal.com/indexd13.htm (am 1.9.2005). Wenn sie im Entscheidungsprozeß im politischen Bereich wären, würden diese Unterschiede wahrscheinlich auch auftreten, die mit Sicherheit nicht zur Lösung der Probleme nicht nur in der Gesellschaft, sondern auch zwischen den Religionen führen würden. 
Zweitens: Frieden wird, wenn überhaupt, durch einen politischen Prozess umgesetzt. Solange die Religionen bei den Entscheidungsprozessen keine Rolle spielen, kann auch kein Weltfrieden von ihnen ausgehen.

Da ein effektiver Dialog zwischen den Kulturen nicht über die Religionen, sondern nur über die Politik stattfinden kann, ist das laizistische System in der Türkei als Mittler geeignet. Der damalige französische Außenminister Hubert Vedrins hat dazu im Februar 2002 auf einer Konferenz in Istanbul sehr treffend bemerkt: »Die beste Antwort auf die These von Huntington - der Kampf der Kulturen ist nicht zu verhindern - ist »Istanbuls Seele «. «24

Der damalige Ministerpräsident Ecevit sagte bei seinem Besuch auf Ground Zero im Januar 2002: »Wenn die Türkei nicht wäre, wäre wahrscheinlich ein Religionskrieg in der Welt ausgelöst worden « ${ }^{25}$, was keineswegs nur ein populistisches Bonmot war.

»Alle Muslime sind vielleicht keine Terroristen, aber alle Terroristen sind Muslime ${ }^{26}$, auf diesen Satz stieß die türkische Journalistin Ferai Tinç bei den Diskussionen in den amerikanischen Medien nach den Terroranschlägen. Die Aussage des italienischen Ministerpräsidenten Berlusconi: »Die westliche Zivilisation ist der islamischen Zivilisation überlegen $\ll^{27}$ und andere ähnliche in der Öffentlichkeit geäußerte Ansichten führen dazu, dass der Kampf zwischen den Kulturen in den Köpfen der Menschen schon längst begonnen hat, so dass sich ein Ministerpräsident in Europa nicht scheut, in der Öffentlichkeit eine solche Aussage zu machen, auch wenn diese in Europa scharf verurteilt wurde. Ein weiterer Beleg dafür ist das folgende Zitat aus einem schwedischen Zeitungsartikel: »Ich fühlte überhaupt nichts, als 100000 Araber starben, habe aber Sympathie für die Soldaten der alliierten Staaten und deren Familien. Ich habe Angst vor Arabern. $\ll^{28}$ Diese Meinung vertrat Erik Hörstadius in der Zeitschrift »Slitz Straux « nach dem ersten Golfkrieg 1991. Die alarmierende Auffassung des ehemaligen schwedischen Botschafters Ingmar Karlsson in Bezug auf diesen Artikel zeigt, welches Niveau die Vorurteile gegen andere Kulturangehörige ereicht haben: »Rassistische Aussagen gegen Araber sind in Schweden wie immer festzustellen. Dieser Artikel erregte, von wenigen Ausnahmen abgesehen, kaum Aufmerksamkeit und löste obendrein auch keine Diskussion in

24 Murat Yetkin, »Issmail Cem >İstanbul Ruhu< İsminden Memnundu (Issmail Cem war mit dem Begriff, Seele Istanbuls zufrieden)« in Radikal, in: Hürriyet, 14.02.2002, (Murat Yetkin ist türkischer Journalist, Cem war türkischer Außenminister und galt als Initiator dieser Konferenz).

25 Hürriyet, 19.01.2002.

26 Ferai Tınç, »Medeniyetler Çatı/masını Türkiye Engeller (Die Türkei kann den Kampf zwischen den Zivilisationen verhindern)« in: Hürriyet, 30.09.2001.

27 Tinç, Medeniyetler Çatı/masını Türkiye Engeller, aaO. (FN 26).

28 Ingmar Karlsson, İslam ve Avrupa, Inanç Ayrllığ - Yafam Birliği (Islam und Europa, unterschiedlicher Glaube - gemeinsames Leben), Originaltitel des Werkes: Islam och Europa Samlevnad eller Konfrontation? Türkçesi (Übersetzung) Gülseren Ergün, Istanbul 1996, S. 11. 
der Gesellschaft aus. Der einfache Grund dafür war, dass die Mehrheit der Bevölkerung in Schweden dasselbe empfand. $\ll^{29}$

Gleiches kann im Übrigen in der islamischen Welt gegen den Westen festgestellt werden. Dass Genugtuung über das tragische Ereignis vom 11. September empfunden werden kann ${ }^{30}$, ist ein anderes Beispiel dafür. Diese Genugtuung ist nicht nur in den islamischen Ländern, sondern auch unter den Muslimen in Mitteleuropa zu beobachten. Der Augsburger Soziologe und Terrorexperte Peter Waldmann untersuchte dieses Phänomen. Interviews des Waldmann-Lehrstuhls mit 60 muslimischen Studenten in ganz Deutschland ergaben folgendes: Diejenigen, die fest in ihr Glaubensmilieu eingebunden sind, fühlen sich selbstsicherer und haben die besseren Studienerfolge. Dagegen äußerten die Muslime, die einen westlichen Lebensstil pflegen, eine gewisse Genugtuung über die Anschläge des 11. September, weil sie die Verwundbarkeit einer Zivilisation aufzeigten, die ihnen selbst immer wieder Enttäuschungen bei ihrem Versuch der Anpassung bereitet. ${ }^{31}$ Dass wegen des Krieges im Irak im März 2003 im Mannheimer und Ulmer Raum, also im Zentrum Europas, laut Erkenntnissen des baden-württembergischen Verfassungsschutzes zum »Heiligen Krieg « aufgerufen worden war ${ }^{32}$, ist vor diesem Hintergrund nicht mehr ganz so erstaunlich.

\section{Priorität der Türkei im Dialog}

Dass der so oft beschworene, tatsächlich aber kaum praktizierte Dialog zwischen Orient und Okzident nach dem Terror vom 11. September notwendiger ist denn je, steht außer Frage. Aber wie kann ein solcher Dialog tatsächlich ins Werk gesetzt werden? Gibt es ein politisches System oder einen Mechanismus, die als Brücke zwischen Osten und Westen fungieren könnten?

Es ist wichtig, auf diese Fragen überzeugende Antworten zu geben, die den Ansprüchen des westlichen politischen und wirtschaftlichen Systems und der östlichen Kultur und des Islam gleichermaßen gerecht werden, zugleich aber doch im Wesentlichen harmonisch koexistieren. Es ist dabei genauso wichtig, dass diese Ansprüche nicht einander zuwiderlaufen, sondern als die von der Mehrheit der Bevölkerung eines Landes freiwillig befürworteten und überzeugend umgesetzten Elemente als Grundlage eines Modells erfolgreich bestehen bleiben.

Mark Parris, von 1997 bis 2000 amerikanischer Botschafter in Ankara, verwies im Rahmen eines Vortrages an der Universität von Virginia in den USA auf das türki-

29 Karlsson, İslam ve Avrupa, İnanç Ayrılı̆g 1 - Yafam Birliği, Originaltitel des Werkes: Islam och Europa Samlevnad eller Konfrontation? Türkçesi Gülseren Ergün, aaO. (FN 28), S. 11.

30 Udo Steinbach, »Islamischer Terrorismus. Ein selbstkritischer >Dialog der Kulturen $<$ ist jetzt gefragt«, in: Internationale Politik, Europa Archiv, Berlin Nr. 3 (2002), S. 7.

31 ohne Verfasser, »Von Religion und Gewalt, Terrorexperte Peter Waldmann nimmt Abschied « in: Allgäuer Zeitung, 30.01.2003.

32 ohne Verfasser, »Auch in Ulm Aufrufe zum >Heiligen Krieg« in: Allgäuer Zeitung, 11.04.2003. 
sche Modell. (Das laizistische System in der Türkei wird im Westen als »türkisches Modell« bezeichnet). Er betonte zusätzlich, die Amerikaner wüssten nicht genug um die Bedeutung der Türkei und fügte hinzu, dass das türkische Modell nach dem 11. September vermehrt vom Westen wahrgenommen wurde. Parris übte auch Kritik an den amerikanischen Medien, die den Besuch des türkischen Ministerpräsidenten Ecevit in den USA im Januar 2002 ignoriert hatten. ${ }^{33}$ Die Überschrift eines Artikels von Tinç in »Hürriyet«: »Die Türkei kann den Kampf zwischen den Zivilisationen verhindern «, unterstreicht die Bedeutung der Türkei und ihres Systems und Tinç argumentiert, dass der beste Dialog zwischen den Religionen in der Türkei besteht. ${ }^{34}$

Dem sind zwei wichtige Punkte hinzuzufügen.

Erstens: Dieser Dialog kann nur im Rahmen des Laizismus stattfinden.

Zweitens: Die Koexistenz der Religionen in der Türkei besteht im Großen und Ganzen erfolgreich, da der islamische Pluralismus durch den Laizismus islamischen bzw. nicht islamischen Gruppen in der Türkei die Möglichkeit bietet, sich zu artikulieren.

Die religiösen Gruppen, unabhängig von ihrer Religionszugehörigkeit, sind gleichberechtigt und bringen ihre Meinung bei aktuellen Anlässen zum Ausdruck. Die Gläubigen, insbesondere Muslime unterschiedlicher religiöser Gruppierungen, werden dadurch über die aktuellen Ereignisse nicht einseitig informiert. Der Verfasser bezeichnet dies als »islamischen Pluralismus«. Die Artikulation im Bereich der Religion ist also eine Voraussetzung. Davon machen auch Personen, die aus dem Islam ausgetreten sind und sich selbst als Atheisten bezeichnen, Gebrauch. Der bekannte türkische Schriftsteller Aziz Nesin und der ehemalige türkische Mufti Turan Dursun vom Religiösen Amt Diyanet sind zwei Beispiele dafür. Dursun übte in der Türkei am Islam recht scharfe Kritik, die in einem anderen Land, dessen Bevölkerung überwiegend islamisch ist, nicht vorstellbar gewesen wäre. So sind »Die satanischen Verse « von Salman Rushdie für ihn die Realität des Islam..$^{35}$ Dursun hat zahlreiche kritische Bücher verfasst, die ohne Zensur in der Türkei erhältlich sind. In diesem Pluralismus haben auch theologische Meinungsverschiedenheiten ihren Platz.

Laizismus in der Türkei führte und führt also dazu, dass keine islamische Gruppe und keine islamischen Wissenschaftler allein die Befugnis zur Interpretation des Korans haben können. Das Gewissen der Gläubigen kann somit durch unterschiedliche Interpretationen, die zum Teil politische Motive haben könnten, nicht einseitig beeinflusst werden. Da der Islam bei der Gesetzgebung in der Türkei keine Rolle spielt, wird die Koexistenz auch unter den islamischen Gruppen fortgesetzt, weil dadurch kein Machtkampf entsteht. Dieser Sachverhalt wird im Übrigen auch in der Türkei in seiner wahren Bedeutung nicht immer hinreichend wahrgenommen. Das Ergebnis ist,

33 Kasım Cindemir, »Mark Parris: Türkiye'nin kıymetini bilmedik (Mark Parris: Wir wussten die Türkei nicht zu schätzen)« in: Hürriyet, 26.01.2002, (Kasım Cindemir ist türkischer Journalist).

34 Tinç, Medeniyetler Çatı/masını Türkiye Engeller, aaO. (FN 26 ).

35 Turan Dursun, Din bu -1-. Tabu Can Çekifiyor (Das ist die Religion - 1 - Das Tabu liegt im Sterben), Istanbul 1994, S 103. 
dass die Türkei durch den religiösen Frieden und die Koexistenz von westlichem politischen System und Islam und von Orient und Okzident eine beispielhafte Dialogmöglichkeit innerhalb der türkischen Grenzen zur Verfügung stellt. Die Bedeutung dieser Möglichkeit nahm nach dem 11. September 2001 aus zwei Gründen noch zu:

Erstens: Die beste Technologie und Aufrüstung sind gegen den Terror machtlos und garantieren keine innere Sicherheit in einem Land. Zweitens: Die USA mussten gegen den von der Religion geprägten Terror kapitulieren. Sie waren trotz ihrer starken Wirtschaft und ihre mächtigen Militärapparats gegen den islamistischen Terror machtlos und müssen nun nach einem anderen, nicht militärischen Weg suchen, den Kampf gegen den religiösen Terror zu gewinnen. Unter diesem Aspekt widerspricht der Verfasser der These des türkischen Wissenschaftlers Osman Metin Öztürk, dass die geographische Abhängigkeit der USA und der anderen westlichen Länder von der Türkei in der neuen Weltordnung nach dem Ende der UdSSR 1991 abnehme ${ }^{36}$, weil das politische Gewicht der Türkei seit dieser Zeit deutlich zugenommen habe; dies zeigte sich bei der Unterstützung des türkischen Systems durch den Westen im Kaukasus und in Zentralasien. ${ }^{37}$ Diese Abhängigkeit des Westens, wenn überhaupt die Rede davon sein kann, erreichte erst recht nach dem 11. September eine ganz andere Dimension. Nach Auffassung des türkischen Journalisten Sedat Ergin war der damalige Ministerpräsident Ecevit bei seinem Besuch in den USA im Januar 2002 bei der amerikanischen Regierung aus diesem Grund angesehener denn je. Der stellvertretende Präsident Dick Cheney hat den Modellcharakter der Türkei unmissverständlich betont: »Die Türkei, die eine stabile islamische Tradition hat, bildet als ein demokratisches und laizistisches Land ein Modell für die ganze islamische Welt. «38 $\mathrm{Ob}$ dieses Modell von den anderen islamischen Ländern in der Zukunft angenommen wird, ist indes mehr als fraglich. Denn diese Länder verfügen nicht über die Erfahrung mit der Säkularisierung, die die Türkei Jahrhunderte zuvor im Osmanischen Reich gesammelt hat. Das Osmanische Reich war keineswegs eine Theokratie, sondern eine Herrschaft, bei der sich die Religion dem Staat klar unterordnete. ${ }^{39}$ Säkulare Komponenten dürfen dabei nicht übersehen werden. ${ }^{40}$ Am 23.12.1876 wurde die erste osmanische Verfassung erlassen. ${ }^{41}$ Diese bildet die

36 Osman Metin Öztürk, »Güneydoğu Sorunu Üzerine (Über die Problematik des Südostens)《 in: Yeni Forum, Aylık Siyaset Iktisat KültürDergisi, Ankara 293 (1993), S. 51.

37 Siehe für Einzelheiten: Necati Iyikan, Die politischen Beziehungen zwischen der Türkei und Aserbaidschan (1992-2003) unter besonderer Berücksichtigung des türkischen Modells im Kaukasus und in Zentralasien und dessen Bedeutung nach den Anschlägen vom 11. September 2001 in den USA, Hamburg 2005, S. 223 - 234, (Necati Iyikan ist türkischer Wissenschaftler).

38 Sedat Ergin, »Ground Zero'dan Türk Modeline (Vom Ground Zero bis zum türkischen Modell)«, in: Hürriyet, 20.01.2002, (Sedat Ergin ist türkischer Journalist).

39 Giacomo Luciani, »Die Türkei und der Islam. Hürde auf dem Weg nach Europa?« in: Internationale Politik, Europa Archiv, Berlin Nr. 3 (2002), S. 29.

40 Klaus Kreiser, »Die Rolle der türkischen Völker « in: Weltmacht Islam, Bayerische Landeszentrale für politische Bildungsarbeit, München 1988, S. 204.

41 Pia Angela Göktürk, Der Werdegang der modernen Türkei. Beiträge zur Geschichte, Gesellschaft, Sprache und Kultur des Landes, Istanbul 1983, S. 10. 
Grundlage für die heutige türkische Republik, denn ab diesem Datum gab es eine demokratische Tendenz. Der Zeitraum von 1876 bis 1908 wird die erste, derjenige von 1908 bis 1918 die zweite konstitutionelle Periode genannt. Die erste osmanische Verfassung, die von Sultan Abdülhamit II. wegen des Krieges zwischen Osmanen und Russen (1877 - 1878) aufgehoben worden war, wurde am 28.07.1908 von Abdülhamit II. erneut in Kraft gesetzt. Danach fanden Wahlen statt. Die Partei für Einheit und Fortschritt hatte die Mehrheit im Parlament, ebenso erhielt die Opposition ein gewisses Gewicht im Parlament. ${ }^{42}$ In diesen Perioden beobachtet man die ersten Schritte zur Demokratisierung. Dies hatte zur Folge, dass die türkische Gesellschaft der 20er- und 30er-Jahre keine traditionelle muslimische Gesellschaft mehr war. Nationalismus und westliches Gedankengut hatten sich längst fest verankert. ${ }^{43} \mathrm{Die}$ Umwandlung des Kernlandes des Osmanischen Reiches in eine Republik durch Kemal Atatürk, die als »der einschneidende Schritt « bezeichnet wurde ${ }^{44}$, beeinflusste nicht nur die Geschichte des Landes, sondern auch den Islam, denn die Gründung der türkischen Republik am 29. Oktober 1923 führte zur Abschaffung des Kalifats ${ }^{45}$ am 3. März 1924 sowie zuletzt zur Einführung des Laizismus 1937.

Das folgende Beispiel dient als Beleg dafür, auf welche Kritik der Laizismus in der Türkei in der islamischen Welt stieß: Scheich Omar Abdul Rahman, ein radikaler islamischer Prediger aus Kairo, rief bei der Mitte Oktober 1986 in Istanbul stattgefundenen internationalen islamischen Konferenz dazu auf, für den Sturz der Regierungen aller Staaten zu kämpfen, die zwar eine muslimische Bevölkerungsmehrheit haben, nicht aber die Scharia, das koranische Gesetz, zur Grundlage hätten. $\mathrm{Zu}$ diesen Staaten gehört seit den Tagen Atatürks auch die Türkei. ${ }^{46}$ Zusätzlich ist die Tatsache zu berücksichtigen, dass nicht nur einzelne Radikale, sondern auch islamische Länder wie der Iran und Saudi-Arabien den Laizismus als antiislamisch einstuften und ihn scharf verurteilten und es heute noch tun. Auch der Faktor, dass Iran und Saudi-Arabien in der laizistischen Türkei propagandistisch und finanziell um die Gunst der Frommen rivalisieren ${ }^{47}$ und vor allem die Absicht Irans, die isla-

42 Celal Aydın, Sosyal Bilimler (Soziale Wissenschaften), Ankara 1987, S. 192, 193.

43 Moser / Weithmann, Die Türkei. Nation zwischen Europa und dem Nahen Osten, aaO. (FN 5), S. 112.

44 Udo Steinbach, »Der Nahostkonflikt im islamischen Horizont « in: Weltmacht Islam, Bayerische Landeszentrale für politische Bildungsarbeit, München 1988, S. 489.

45 Das Abschaffen des Kalifates wurde von einem Teil der Presse in Istanbul folgendermaßen kritisiert: »Wenn wir das Kalifat nicht mehr haben, hat das Land bei den islamischen Ländern kein Gewicht mehr.« Mustafa Kemal Atatürk, Nutuk (Die Rede), Ankara 2002, S. 125.

46 Scheich Omar hatte mit seiner »Fatwa « - einem sich auf den Koran beziehenden »Freibrief « - den Mördern des ägyptischen Staatspräsidenten Sadat im Vorgriff auf die Bluttat die Absolution erteilt. Nach dem Attentat wurde er zwar verhaftet und angeklagt, später jedoch von den ägyptischen Behörden wieder freigelassen. Rainer Werle / Renate Kreile, Renaissance des Islam. Das Beispiel Türkei, Hamburg 1987, S. 113.

47 Wolfgang Günter Lerch, »Angst in den Palästen. Saudi-Arabien und die Golfemirate« in: Peter Scholl-Latour, Weltkrise Arabien. Allah, Blut und Öl-Hintergründe eines Konflikts, Gütersloh 1992, S. 1. 
mische Bewegung im Iran in die Türkei zu exportieren, so meint Eygi ${ }^{4}$, sind wichtige Zeichen dafür, dass die Einführung des türkischen Systems in anderen islamischen Ländern zumindest in absehbarer Zeit ein unerfüllbarer Wunsch bleiben wird.

\subsection{Konferenz zum Zweck des Dialogs}

Die ausschlaggebende Frage jedoch, ob die Türkei geeignet wäre, den Dialog zwischen der christlichen und islamischen Welt $\mathrm{zu}$ initiieren, ist mit einem klaren » $\mathrm{a}$ « zu beantworten. Ein überzeugendes Beispiel mag die Konferenz am 13.02.2002 in Istanbul sein, deren Ziel es war, die christliche und die islamische Welt zum ersten Mal nach den Terroranschlägen vom 11. September 2001 zusammenzubringen. Diese fand auf Initiative der Türkei statt. Die Zahl der daran teilnehmenden Länder war mit 74 bemerkenswert hoch. ${ }^{49} 51$ Minister und 74 Repräsentanten aus den USA, Europa und der Organisation der islamischen Konferenz waren dabei anwesend. ${ }^{50}$ Diese hohe Beteiligung kann man nicht nur mit dem Organisationstalent des türkischen Außenministeriums erklären. Der wahre Grund dafür lag vielmehr in einem sowohl auf islamischer als auch auf christlicher Seite zunehmenden Bedürfnis nach einem solchen Dialog. Das Presseecho auf diese Konferenz in Istanbul war der Bedeutung angemessen wie zahllose Zeitungsschlagzeilen belegen. ${ }^{51}$

48 Aysel Ekfi, Din Devletleri (Die theokratischen Staaten), Ankara 1995, S. 21, (Aysel Ekfi ist Dekanin der Juristischen Fakultät, Istanbul). Mehmet ${ }^{\mathrm{TM}}$ evki Eygi, der im religiösen Bereich in der Türkei bekannt ist, äußerte sich gegenüber der Tageszeitung Hürriyet. Hürriyet, 12.03.1995. Das Werk der türkischen Wissenschaftlerin besteht aus den Aufsatzsammlungen verschiedener Zeitungen.

49 Okay Gönensin, »11. Eylül Türkiye Için Önemli Dönüm Noktasıdır (Der 11. September bildet eine wichtige Wende für die Türkei)« in: Sabah, in: Hürriyet, 14.02.2002, (Okay Göneṇsin ist türkischer Journalist).

50 Ali Sirmen, »Istanbul Ruhu ve Kültürlerarası Diyalog (Seele Istanbuls und Dialog zwischen den Kulturen), in: Cumhuriyet, in: Hürriyet, 15.02.2002, (Ali Sirmen ist türkischer Journalist).

51 »Bin Ladin hat zerstört, Cem hat aufgebaut « (Cem war türkischer Außenminister und galt als der Initiator dieser Konferenz); Der nach dem 11. September zwischen der islamischen und christlichen Welt wehende kalte Sturm hat sich gelegt (Milliyet, 13.02.2002); »Der Tanz der Zivilisationen«. Der Westen und der Osten haben am ersten Tag dieses Spitzentreffens zueinander Stellung bezogen: Europa hat die Stelle der demokratischen Werte im Islam thematisiert. Die islamischen Länder haben auf die Notwendigkeit einer für alle gültige Definition von »Terror« aufmerksam gemacht. (Radikal, 13.02.2002); "Die EU und die islamischen Länder sind sich darüber einig: Die größte Gefahr ist der Terror « (Cumburiyet, 13.02.2002); "So wird ein führender Staat « Die Türkei hat die westlichen und östlichen Zivilisationen, die nach dem 11. September aneinander gezweifelt hatten, zum ersten Mal bei einem Spitzentreffen in Istanbul zusammengebracht. Die Türkei hat dadurch als »Brücke« zwischen Westen und Osten gedient. (Sabah, 13.02.2002); »Wir wurden die Brücke zum Frieden« Die Kulturen im Osten und Westen haben unter der Führung der Türkei den Frieden gesucht. Sie trafen sich in der Geschichte in dieser großer Beteiligung zum ersten Mal und beschlossen die 
Der französische Außenminister Hubert Vedrin zeigte sich überzeugt: »Istanbuls Seele wird dazu führen, dass der Dialog zwischen den islamischen und europäischen Ländern eröffnet wird « und Prinz Hassan Bin Tallal von Jordanien meinte: »Der Westen und der Osten müssen sich verstehen, damit Istanbuls Seele Erfolg haben kann. ${ }^{52}$ Die Aussage des jordanischen Prinzen unterstreicht die Bedeutung dieser Konferenz für den Eintritt in einen wirklichen Dialog: »Wir treffen uns auch bei den UNO-Sitzungen. Jeder liest dort aber nur die offizielle Erklärung seiner Regierung zu aktuellen Angelegenheiten vor. Das ist kein Dialog. Wir hatten in Istanbul zum ersten Mal die Möglichkeit, nicht aneinander vorbei, sondern miteinander zu sprechen « und Deutschlands Außenminister Joschka Fischer definierte den Begriff »Dia$\log$ « wie folgt: » Dialog bedeutet nicht, miteinander höflich umzugehen und nur leere Worte auszusprechen. Wir müssen aufhören, uns gegenseitig zu belehren. Die Zeit, in der wir voneinander etwas lernen, ist gekommen. $\aleph^{53}$ Fischers Ansicht war ein wichtiges Zeichen dafür, dass der 11. September eine Wende für die Zukunft des Dialogs in der Welt markierte und diese Wende von den politischen Akteuren der Welt auch ernst- und wahrgenommen wurde. Die bereits erwähnte Feststellung Vedrins, die den Dialog bei dieser Konferenz mit der These Huntingtons, der Kampf der Kulturen sei nicht $\mathrm{zu}$ verhindern, in Zusammenhang bringt und als eine Antwort darauf sieht ${ }^{54}$, ist nicht nur eine Zusammenfassung der Konferenz, sondern zugleich ein Hinweis auf die herausragende Rolle der Türkei mit ihrem laizistischen System. Nach Okay Gönensin war der 11. September ein Wendepunkt auch für die Türkei. Die Bedeutung der Türkei und ihres Modells stießen auf besseres Verständnis. ${ }^{55}$ Nach Ali Sirmen bildete »Istanbuls Seele« einen guten Anfang für den Dialog zwischen den Kulturen. ${ }^{56}$

Das nächste ausführliche Beispiel ist aus dem Alltag gegriffen und erweckt im ersten Moment vielleicht den Eindruck, als wäre es von keiner großen Bedeutung. Es ist jedoch ein weiterer überzeugender Hinweis darauf, dass die Türkei nicht nur durch ihr System, sondern auch durch den erreichten Frieden und die Toleranz zwischen den verschiedenen Religionen und Kulturen im Alltag ein dialogfähiges Land ist. Der türkische Intellektuelle Özdemir Ince verfasste einen umfangreichen Bericht über die Bedeutung eines Friedhofes, der in der südlichen Stadt Mersin in der Türkei liegt. Die Überschrift lautete: »Der die Theorie von Huntington >Kampf der Kulturen seit 63 Jahren dementierende Friedhof. « ${ }^{57}$ Dieser Friedhof hat eine Größe

51 Zusammenarbeit, um eine lebenswürdige Erde zu ermöglichen. (Türkiye, 13.02.2002); »Der Dialog wurde notwendig « Zeitpunkt und Ort dieses Treffens haben eine historische Bedeutung. Der internationale Dialog ist keine Alternative mehr, sondern Notwendigkeit. (Zaman, 13.02 2002); »Türkei, du bist die Größte!« Die ganze Welt beklatscht die Türkei, die die Staatsmänner aus 74 Ländern in Istanbul zusammenbrachte. Ankara baute die Brücke zwischen Osten und Westen bei dieser wichtigen Organisation auf, womit sie sehr an Prestige gewann. (Posta, 13.02.2002).

52 Hürriyet, 13.02.2002.

53 Yetkin, Ịsmail Cem >İstanbul Ruhu< İsminden Memnundu, aaO. (FN 24).

54 Yetkin, İsmail Cem Iİstanbul Ruhu< İsminden Memnundu, aaO. (FN 24).

55 Gönensin, 11. Eylül Türkiye İçin Önemli Dönüm Noktasıdır, aaO. (FN 49).

56 Sirmen, Istanbul Ruhu ve Kültürlerarası Diyalog, aaO. (FN 50).

57 Ince Özdemir, »Üç Dinden Ortak Mesaj (Die gleiche Botschaft von drei Religionen)« in: Hürriyet, 28.10.2001. 
von ca. $350000 \mathrm{~m}^{2}$. Die Zahl der verschiedenen Religionsangehörigen im Jahre 1930 berücksichtigend, hatten die Muslime $175000 \mathrm{~m}^{2}$ und die Juden und die Christen ebenfalls $175000 \mathrm{~m}^{2}$ erhalten. Die Zahl der Muslime nahm mit der Zeit zu, während die Zahl der Juden und Christen abnahm. Das führte dazu, dass die am Anfang beabsichtigte Grenze zwischen den verschiedenen Gläubigen nicht mehr existierte. Ihre Aufhebung wurde vom damaligen Oberbürgermeister Mithat Toroglu und dem Stadtrat bestätigt. Dies ist die amtliche Seite der Toleranz. Die drei Religionen haben keine reservierten Plätze mehr auf diesem Friedhof. Die verstorbenen Angehörigen der Religionen sind deswegen dicht nebeneinander beigesetzt. Die Bevölkerung in dieser Stadt hält das für völlig natürlich. Nach Ince hätten die einheimischen Muslime ihre Verstorbenen woanders beigesetzt, wenn sie damit nicht einverstanden gewesen wären. ${ }^{58}$ Das ist die andere Seite der Toleranz, nämlich die der Bevölkerung. Der türkische Wissenschaftler Uğur Ersoy, Enkel des damaligen Muftis Abdullah Siddik Efendi, berichtet, dass eben jener versicherte, diese Art eines Friedhofs sei aus islamischer Perspektive erlaubt. ${ }^{59}$ Das ist der Hinweis auf die islamische Toleranz. »Dieser Friedhof ist ein Symbol für den Frieden«, sagte dessen Direktor Recai Apaydın im Jahr 2001. Der zu der Zeit amtierende Mufti Mazhar Bilgin wünschte sich, dass der Friedhof in der heutigen Zeit, in der Terror, Kriege, Blutvergießen auf der Tagesordnung sind, ein Beispiel für die Toleranz werde. ${ }^{60}$ Der Pfarrer der katholischen Gemeinde, Gregorio B. Simonelle, unterstrich, dass der Friedhof ein Beispiel für andere Friedhöfe werden sollte: »Dieser Friedhof verbindet nicht nur die Andersgläubigen, sondern auch die Christen wie römisch-katholische, maronitische, syrische und chaldäische Christen. Auch ich habe für mich einen Platz auf diesem Friedhof reserviert. «61 Das ist ein weiteres Zeichen für Toleranz, derjenigen der christlichen Seite.

Hätte die Türkei das kühne Angebot aus Washington angenommen, als einziges muslimisches NATO-Land den USA dabei zu helfen Saddam Hussein aus dem Amt zu bomben und sich als Mitgift dafür an den nordirakischen Ölquellen von Kirkuk und Mosul zu bedienen ${ }^{62}$, hätte die Besonderheit des Systems keine Rolle mehr gespielt. Erinnert man sich auch der Sorge König Hassans von Jordanien vor dem ersten Golfkrieg 1990, dass der die arabische Welt in eine Krise nie gekanntem Ausmaßes stürzen würde ${ }^{63}$, muss Ankara weiterhin in der krisenreichen Region eine sehr vorsichtige Politik verfolgen, wenn sie die Brücken- oder Dialogfunktion zwischen Okzident und Orient wahrnehmen will. Diese Funktion kann sie nur deshalb weiterhin wahrnehmen, weil Ankara die aktive Beteiligung am Krieg gegen den Irak im März 2003 verweigert hat. Ansonsten hätte die Türkei ihre Glaubwürdigkeit in der islamischen Welt verloren. Hasan Hanefi, Philosophieprofessor an der

58 Özdemir, Üç Dinden Ortak Mesaj, aaO. (FN 57).

59 Özdemir, Üç Dinden Ortak Mesaj, aaO. (FN 57).

60 Özdemir, Üç Dinden Ortak Mesaj, aaO. (FN 57).

61 Özdemir, Üç Dinden Ortak Mesaj, aaO. (FN 57).

62 Christiane Schlötzer, »Anmache in Ankara« in: Süddeutsche Zeitung, 01.12.2001.

63 Wolfgang Günter Lerch, Kein Frieden für Allabs Völker. Die Kriege am Golf. Geschichte, Gestalten, Folgen, Frankfurt am Main 1991, S. 176. 
Universität Kairo in Ägypten, befand denn auch auf die vor dem Krieg gegen den Irak gestellte Frage, ob so eine Konferenz wieder in der Türkei stattfinden könne, wenn die Türkei bei einer möglichen Operation gegen den Irak an der Seite der USA stünde: »Niemals! «.6 ${ }^{64}$ Das Parlament in Ankara lehnte am 1. März 2003 ab, die türkische Grenze zum Irak für die 62.000 amerikanischen Soldaten zu öffnen. ${ }^{65}$ Diese Entscheidung hat die politische Führung in Washington überrascht und verärgert. Die Rolle des türkischen Systems als Brücke zwischen den Kulturen wurde dadurch jedoch gestärkt.

\subsection{Erfolg des Laizismus in der Türkei}

Das laizistische System in der Türkei kann einen großen, in der islamischen Geschichte bisher nicht da gewesenen Erfolg vorweisen. Dieser heißt »Integration einer islamischen Partei in das laizistische System und die Demokratisierung des politischen Islam«. Recep Tayyip Erdoğan ist die Person, die dabei die entscheidende Rolle spielen kann. Er hatte im August 2001 die Partei AKP gegründet, die bei der vorgezogenen Parlamentswahl am 3. November 2002 auf rund 35 Prozent der abgegebenen Stimmen $\mathrm{kam}^{66}$, womit er, bedingt durch das Wahlsystem, mit 65 Prozent die absolute Mehrheit im Parlament errang. Bei den Parlamentswahlen 2007 erreichte die AKP unter seiner Führung mit 46,6 Prozent der Wählerstimmen 340 Sitze im Parlament und damit abermals die absolute Mehrheit im Parlament mit $61,8 \% .{ }^{67}$

Der Werdegang des Sohnes frommer Zuwanderer aus dem Schwarzmeergebiet hat im Armenviertel Kasimpasa a in Istanbul begonnen. Der spätere Unternehmer ist vom Besuch der religiösen Imam-Hatip-Schule, an der Prediger und Vorbeter ausgebildet werden, sowie von Necmeddin Erbakan, der grauen Eminenz des politischen Islam in der Türkei, geprägt. Erbakan lehnte als Vorsitzender der Nationalen Heilspartei MSP68 in den siebziger Jahren alle vertraglichen Bindungen der Türkei mit der westlichen Welt ab. Stattdessen wollte er einen gemeinsamen Wirtschaftsmarkt mit den arabischen Ländern aufbauen. ${ }^{69}$ Er drohte: »Der islamische Staat muss kommen, nur ist nicht sicher, ob dieser Durchbruch mit Schweiß oder mit Blut errungen wird. «70 Erdogan wurde im Alter von 40 Jahren als Mitglied

64 Sirmen, İstanbul Ruhu ve Kültürlerarası Diyalog, aaO. (FN 50).

65 TRT Telegün, 01.03.2003.

66 Allgäuer Zeitung, 04.11.2002.

67 http://www.radikal.com.tr/haber.php?haberno=227671\&tarih=23/07/2007 http://www.inankara.org/haberler/haberincele.php?haberid=Njcy

68 Diese Partei war bis zum Putsch am 12. September 1980 wegen ihrer antilaizistischen Aktivitäten auffällig. Sie wurde zusammen mit anderen Parteien von den Putschisten verboten.

69 Faruk ${ }^{\mathrm{TM}} \mathrm{en}$, IIslamischer Fundamentalismus und die türkische Minderheit in der Bundesrepublik Deutschland « in: Thomas Meyer, Fundamentalismus in der modernen Welt. Die Internationale der Vernunft. Frankfurt am Main 1989, S. 296.

70 Peter Scholl-Latour, Allabs Schatten über Atatürk. Die Türkei in der Zerreißprobe, Berlin 1999, S. 195. 
der Wohlfahrtspartei (RP), der Nachfolgerin der verbotenen MSP, zum Oberbürgermeister von Istanbul gewählt. Sein Amt verlor er nach seiner Verurteilung wegen »religiöser Aufwiegelung « im April 1998 und verbrachte vier Monate der zehnmonatigen Haftstrafe im Gefängnis. ${ }^{71}$ Es stellt sich die Frage, welches Ziel er nun anstreben wird, da er die Mehrheit im Parlament hat. Erdoğan sagte vor Jahren, als er noch gegen den laizistischen Staat wetterte: »Die Demokratie ist wie eine Straßenbahn - ein Vehikel, das einen zum Ziel bringt. « ${ }^{72}$ Zunächst vermittelte er den Eindruck, eine islamische Ordnung ausrufen zu wollen. Tatsächlich aber verfolgt er eine völlig andere Politik. Er bemüht sich um die beschleunigte Annährung der Türkei an die EU. Die mögliche Mitgliedschaft der Türkei in der EU hätte zur Folge, dass eine Islamisierung der Türkei nicht mehr in Frage kommt. Wenn die Türkei die notwendigen politischen und wirtschaftlichen Reformen unter einem ehemaligen Islamisten wie Erdogan vollziehen könnte, hätte das laizistische System eine islamische Partei voll integriert. Dies wäre ein einmaliges Beispiel in der islamischen Geschichte. Erdoğan beteuert, dass seine Partei AKP so etwas wie eine islamische Version der deutschen Christlich Demokratischen Union (CDU) und kein Verein für Gotteskrieger sei. ${ }^{73}$ Diese Entwicklung wird im Westen in einer Zeit, in der der religiöse Terror im Vordergrund steht, kaum wahrgenommen. Dieser Erfolg der laizistischen Türkei könnte sich jedoch in der Zukunft als eine vielversprechende Perspektive im Kampf gegen den islamischen Fundamentalismus erweisen.

Die eventuelle EU-Mitgliedschaft der Türkei, die schon als das Ende der Europäischen Union bezeichnet wurde ${ }^{74}$, könnte mittlefristig auch den Anstoß zu grundlegenden politischen Umstrukturierungen in den Nachbarländern wie Iran und Syrien geben, denn es ist kaum denkbar, dass diese Länder mit der Europäischen Union von dann 28 Mitgliedsländern mit geschätzten 600 Millionen Einwohnern eine gemeinsame Grenze haben und trotzdem ihre totalitären und fundamentalistischen Regime fortsetzen könnten. Dieser Weg erscheint jedenfalls aussichtsreicher als militärische Interventionen zur Installation einer demokratischen Ordnung nach westlichem Vorbild. Auch vor diesem Hintegrund sollte die EU dem von der Türkei bereits am 14. April 1987 gestellten Aufnahmeantrag in die EU möglichst bald stattgeben. Die Türkei ist mit ihrem Modell als effektiver Dialogpartner zwischen der islamischen und christlichen Welt unverzichtbar.

71 Washington Post, 22.04.1998.

72 Allgäuer Zeitung, 05.11.2002.

73 Süddeutsche Zeitung, 02./03.11.2002.

74 Ohne Verfasser, »Präsident des Verfassungskonvents sorgt für Wirbel. `Ein Beitritt der Türkei wäre das Ende der EU< « in: Süddeutsche Zeitung, 09./10.10.2002. Diese Meinung wurde von einer so wichtigen Persönlichkeit wie Valery Giscard D'Estaing, dem Präsidenten des EU-Reformkonvents und ehemaligen Staatspräsidenten von Frankreich, zum Ausdruck gebracht. 


\section{Zusammenfassung}

Die Ereignisse vom 11. September 2001 in den USA sind nicht nur für die Sicherheit der USA, sondern auch für die Rolle der laizistischen Türkei als Moderator eines Dialogs zwischen der islamischen und christlichen Welt von enormer Bedeutung.

Die mächtige militärische Aufrüstung, moderne Kommunikationsmöglichkeiten und die hohe Technologie der USA konnten den Terroranschlag am 11. September 2001 nicht verhindern. Religiös motivierter Terror lässt sich denn auch mit militärischen Mitteln allein nicht wirksam bekämpfen.

Die Türkei kann als Erfolg für sich verbuchen, dass es ihr trotz langjährig bestehender sozialer, wirtschaftlicher und politischer Problemen gelungen ist, die Ansprüche des westlichen Systems wie auch der islamischen Kultur zu erfüllen und sie im Wesentlichen in Einklang zu bringen.

\section{Summary}

September 11 events in 2001 are particularly important for not only the safety of the USA but the dialogue leadership of secular Turkish Republic in Islamic and Christian world.

Powerful military equipment, modern communication facilities and advanced technology of the USA failed to prevent September 11 terrorism. It has been evident that religious terrorism can not be eradicated with military facilities.

Despite the prevailing social, economic and political problems, Turkey managed to fulfil the requirements of Western system and Islamic culture and adapted to both in general sense.

Necati Iyikan, The events of September 11th 2001's political importance for Turkey 